MSUHEP-041221

MCTP-04-74

\title{
Virtual effects of light gauginos and higgsinos: a precision electroweak analysis of split supersymmetry
}

\author{
Stephen P. Martin ${ }^{a}$, Kazuhiro Tobe ${ }^{b}$, James D. Wells ${ }^{c}$ \\ (a) Physics Department, Northern Illinois University, DeKalb, IL 60115 \\ and Fermi National Accelerator Laboratory, PO Box 500, Batavia, IL 60510 \\ ${ }^{(b)}$ Department of Physics and Astronomy \\ Michigan State University, East Lansing, MI 48824 \\ ${ }^{(c)}$ Michigan Center for Theoretical Physics (MCTP) \\ University of Michigan, Ann Arbor, MI 48109-1120, USA
}

\begin{abstract}
We compute corrections to precision electroweak observables in supersymmetry in the limit that scalar superpartners are very massive and decoupled. This leaves charginos and neutralinos and a Standard Model-like Higgs boson as the only states with unknown mass substantially affecting the analysis. We give complete formulas for the chargino and neutralino contributions, derive simple analytic results for the pure gaugino and higgsino cases, and study the general case. We find that in all circumstances, the precision electroweak fit improves when the charginos and neutralinos are near the current direct limits. Larger higgsino and gaugino masses worsen the fit as the theory predictions asymptotically approach those of the Standard Model. Since the Standard Model is considered by most to be an adequate fit to the precision electroweak data, an important corollary to our analysis is that all regions of parameter space allowed by direct collider constraints are also allowed by precision electroweak constraints in split supersymmetry.
\end{abstract}

hep-ph/0412424

December 2004 


\section{Introduction}

Ordinary intuition about finetuning and naturalness applied to supersymmetry implies the existence of light ( $\mathrm{TeV}$ or less) scalar superpartners, gauginos and higgsinos. Supersymmetry has several other good reasons for its existence beyond its ability to naturally stabilize the weak scale and high scale (e.g., GUT scale or Planck scale). For example, supersymmetry is nicely compatible with gauge coupling unification and dark matter. These two good reasons are arguably less philosophical than the naturalness reason.

There has been much discussion recently centered on supplanting [1] or suspending [2] our ordinary view of naturalness from consideration in supersymmetry model building to allow for very heavy scalar masses. A large hierarchy between scalars and fermions is sometimes called split supersymmetry [3]. The phenomenology of split supersymmetry, where all scalars (even third generation) are significantly heavier than the gaugino masses, has unique features that put it in contrast with other approaches to supersymmetry (see also [4, 5]). The ideas of split supersymmetry may have interesting motivations within string theory [6].

In this article, we wish to study the effects of light gauginos and higgsinos on precision electroweak analysis. We will demonstrate below that the best fit to the precision electroweak data, when the scalar superpartners are decoupled, is light gauginos and higgsinos near the current direct collider limits. As a corollary to this finding, no combination of gaugino and higgsino masses above the current direct experimental limits are in conflict with the precision electroweak data. This is because as the gauginos and higgsino get heavier, the fit approaches the Standard Model fit, which is known to be compatible with the data as long as the Higgs mass is lighter than about $200 \mathrm{GeV}$. Such a cap on the Higgs boson is guaranteed in minimal supersymmetry even if the superpartner masses decouple to the grand unification scale.

We start our analysis by computing the $\chi^{2}$ fit to the precision data within the Standard Model using the latest data and theoretical computations of observables. We then review the corrections to the precision observables for general beyond-the-SM contributions to vector boson self-energies. We also provide explicit formulas for these self-energy functions in the general supersymmetric version of the Standard Model [7, 8]. This is then specialized to our case of light gauginos/higgsinos and heavy scalar superpartners. In the process, we provide some useful analytic results in the pure gaugino and in the pure higgsino limits. The key task within these sections is to justify the claims made above. These results and additional thoughts are summarized in the conclusions section. 


\section{Standard Model precision electroweak fit}

The Higgs scalar boson has not been found by direct experiment, yet its effects are present in precision electroweak observables by virtue of its contributions at one loop to the self energies of electroweak vector bosons. In general, new particles that affect observables only through their induced oblique corrections, such as the Higgs boson, are best constrained [9] by the three observables $\sin ^{2} \theta_{\text {eff }}^{l}\left(=s_{\text {eff }}^{2}\right), M_{W}$ and $\Gamma\left(Z \rightarrow l^{+} l^{-}\right)\left(=\Gamma_{l}\right)$.

The measurements of these three observables [10, 11] are

$$
\begin{aligned}
s_{\text {eff }}^{2} & =0.23147 \pm 0.00017[\text { Average of all results] } \\
M_{W} & =80.425 \pm 0.034 \mathrm{GeV} \\
\Gamma_{l} & =83.984 \pm 0.086 \mathrm{MeV} .
\end{aligned}
$$

It should be noted that the $s_{\text {eff }}^{2}$ determined by the hadronic asymmetries and the one determined by the leptonic asymmetries have a $2.8 \sigma$ discrepancy:

$$
\begin{aligned}
& s_{\text {eff }}^{2}=0.23113 \pm 0.00021\left[A_{F B}^{0, l}, A_{l}\left(P_{\tau}\right), A_{l}(S L D)\right] \\
& s_{\text {eff }}^{2}=0.23213 \pm 0.00029\left[A_{F B}^{0, b}, A_{F B}^{0, c},\left\langle Q_{F B}\right\rangle\right]
\end{aligned}
$$

For the Standard Model (SM) analysis, we use the state-of-the-art computations for $s_{\text {eff }}^{2}$ [12], $M_{W}$ [13] and $\Gamma_{l}$ [14. In the SM, these are expressed in terms of the SM parameters, $\Delta \alpha_{h}^{(5)}\left(M_{Z}\right), M_{t}, \alpha_{s}\left(M_{Z}\right)$ and $M_{h}$. For the experimental values of the SM parameters, we employ the following values:

$$
\begin{aligned}
\Delta \alpha_{h}^{(5)}\left(M_{Z}\right) & =0.02769 \pm 0.00035[15] \\
M_{t} & =178.0 \pm 4.3 \mathrm{GeV}[16] \\
\alpha_{s}\left(M_{Z}\right) & =0.1187 \pm 0.0020[17]
\end{aligned}
$$

In our analysis, $M_{Z}$ is fixed to be $91.1875 \mathrm{GeV}$.

By searching for the minimum of $\chi^{2}$ for the electroweak observables, the best-fit Higgs mass can be found. This is carried out by computing $\chi^{2}\left(M_{h}, M_{t}, \alpha_{s}, \Delta \alpha_{h}^{(5)}\right)=\sum_{X}(X-$ $\left.X_{\exp }\right)^{2} / \sigma_{X}^{2}$ where $X=\left(M_{t}, \alpha_{s}, \Delta \alpha_{h}^{(5)}, s_{\text {eff }}^{2}, M_{W}, \Gamma_{l}\right)$, with the last three predicted in terms of the first four by their Standard Model expressions, and $X_{\exp }, \sigma_{X}$ the experimental central values and uncertainties. In fig. 1, the minimum total $\chi^{2}$ in the SM is shown as a function of $M_{h}$, with the best-fit values given in Table 1. In all cases, the best fit is achieved for $M_{W}$ and $s_{\text {eff }}^{2}$ lower than their experimental central values, and $\Gamma_{l}$ higher than its experimental central value. 


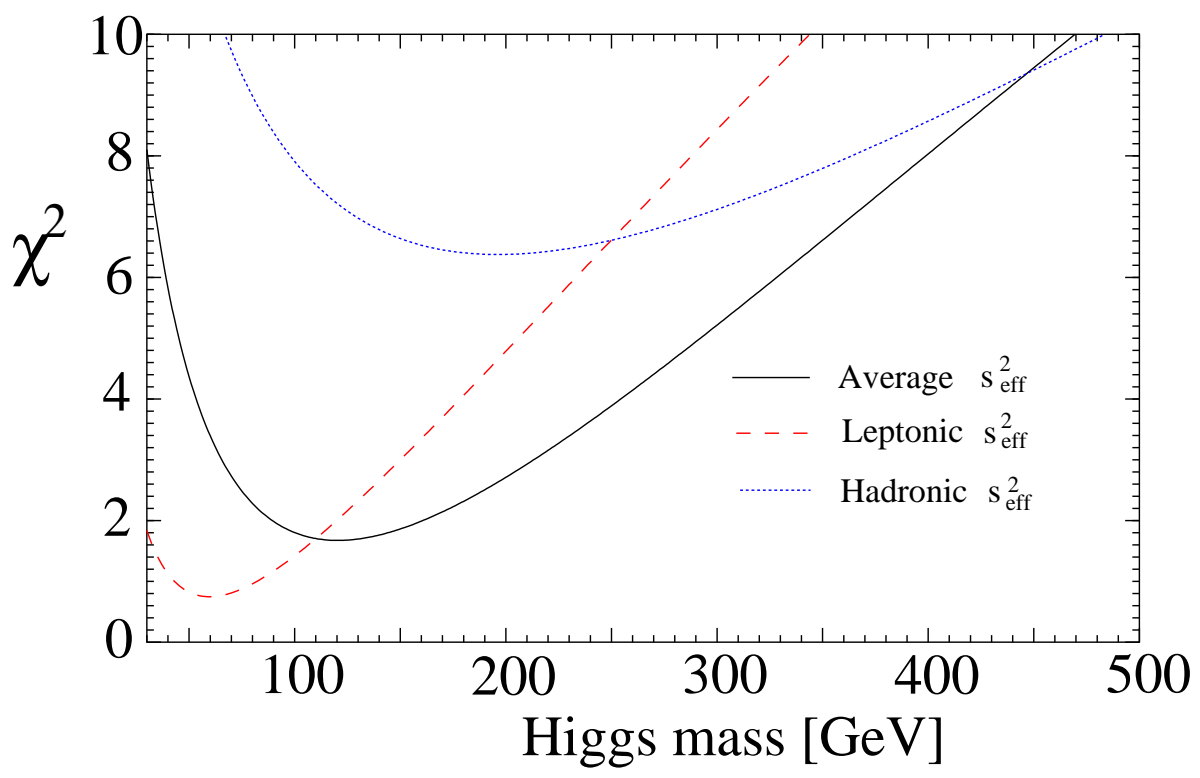

Figure 1: Minimum total $\chi^{2}$ as a function of $M_{h}$ using different values of $s_{\text {eff }}^{2}$ : the average $s_{\text {eff }}^{2}=0.23147 \pm 0.00017$ (solid line), the one from leptonic processes only $s_{\text {eff }}^{2}=0.23113 \pm$ 0.00021 (dashed line), and the one from hadronic processes only $s_{\text {eff }}^{2}=0.23213 \pm 0.00029$ (dotted line).

The minimum of the $\chi^{2}$ is at $M_{h}=120 \mathrm{GeV}$ if the total averaged value of $s_{\text {eff }}^{2}$ is used in the analysis. The $95 \%$ confidence level upper bound on the Higgs mass, which requires $\Delta \chi^{2}<1.64$, comes to $227 \mathrm{GeV}$. Note that if we used only the leptonic data to compute $s_{\text {eff }}^{2}$, the precision fit would have given a best fit value for $M_{h}$ of $60 \mathrm{GeV}$ and a $95 \%$ CL upper bound of $132 \mathrm{GeV}$. If we used only the hadronic data to compute $s_{\text {eff }}^{2}$ we obtain a much lower quality fit, with $M_{h}$ of $196 \mathrm{GeV}$, and a $95 \%$ CL upper bound of $365 \mathrm{GeV}$.

Although these differences in the Higgs mass fit between the leptonic- and hadronic-

\begin{tabular}{|c|c|c|c|c|c|c|c|}
\hline$\chi^{2}$ & $M_{h}[\mathrm{GeV}]$ & $M_{t}[\mathrm{GeV}]$ & $\alpha_{S}\left(M_{Z}\right)$ & $\Delta \alpha_{\text {had }}^{(5)}\left(M_{Z}\right)$ & $M_{W}[\mathrm{GeV}]$ & $s_{\text {eff }}^{2}$ & $\Gamma_{l}[\mathrm{MeV}]$ \\
\hline \hline 1.67 & 120 & 178.40 & 0.1187 & 0.02775 & 80.391 & 0.23140 & 84.043 \\
& & $(+0.1 \sigma)$ & $(0 \sigma)$ & $(+0.2 \sigma)$ & $(-1.0 \sigma)$ & $(-0.4 \sigma)$ & $(+0.7 \sigma)$ \\
\hline 0.75 & 60 & 176.81 & 0.1187 & 0.02772 & 80.418 & 0.23111 & 84.052 \\
& & $(-0.3 \sigma)$ & $(0 \sigma)$ & $(+0.1 \sigma)$ & $(-0.2 \sigma)$ & $(-0.1 \sigma)$ & $(+0.8 \sigma)$ \\
\hline 6.38 & 196 & 179.46 & 0.1187 & 0.02784 & 80.366 & 0.23163 & 84.014 \\
& & $(+0.3 \sigma)$ & $(0 \sigma)$ & $(+0.4 \sigma)$ & $(-1.7 \sigma)$ & $(-1.7 \sigma)$ & $(+0.4 \sigma)$ \\
\hline
\end{tabular}

Table 1: Results of the best fit for the electroweak observables in the Standard Model. The first, second, and third rows use the averaged, leptonic, and hadronic values for $s_{\text {eff }}^{2}$, respectively. 
determined $s_{\text {eff }}^{2}$ are interesting, our view at the present is that we should not differentiate the data, so we take the world averaged $s_{\text {eff }}^{2}$ as the appropriate observable in our analysis of the Standard Model and its extensions.

\section{Oblique corrections to electroweak observables}

Throughout this paper we will be working in a theoretical framework where no corrections to electroweak observables are expected through vertex loops. Such an assumption is valid for many theories. No vertex loop corrections are important if all flavor-charged states that would have contributed to the vertex corrections are too massive to be relevant. In this case, all substantive corrections come from loop corrections of the vector boson self-energies, which only requires the presence of light states charged under the symmetries these bosons generate.

In our case, the flavor-charged squark and slepton fields are decoupled in split supersymmetry, suppressing vertex corrections. However, the gauge-charged charginos and neutralinos are not decoupled and can contribute substantively to the electroweak boson self-energies. That is why we focus on the oblique corrections.

In this section we give the formalism for general oblique corrections. We then apply this formalism to the charginos and neutralinos of minimal supersymmetry. We also briefly describe heavy scalar corrections to the oblique corrections, which will be helpful in characterizing the small corrections to the chargino/neutralino results from the heavy scalars sector.

\section{General oblique corrections}

Our analysis uses the $S, T, U$ parameter expansions of [18], augmented by $Y, V, W$ parameters inspired by [19]. The latter take into account the corrections from nonzero momentum that are important when the new physics states have mass near $M_{Z}$. This is crucial in particular for light charginos and neutralinos in the MSSM, and will become more important in the future when the top-quark mass, the $W$ boson mass, and other electroweak observables become known with better accuracy.

The $S, T, U$ Peskin-Takeuchi parameters are defined as

$$
\begin{aligned}
\frac{\alpha S}{4 s_{W}^{2} c_{W}^{2}} & =\frac{\Pi_{Z Z}\left(M_{Z}^{2}\right)-\Pi_{Z Z}(0)}{M_{Z}^{2}}-\frac{c_{2 W}}{c_{W} s_{W}} \frac{\Pi_{Z \gamma}\left(M_{Z}^{2}\right)}{M_{Z}^{2}}-\frac{\Pi_{\gamma \gamma}\left(M_{Z}^{2}\right)}{M_{Z}^{2}} \\
\alpha T & =\frac{\Pi_{W W}(0)}{M_{W}^{2}}-\frac{\Pi_{Z Z}(0)}{M_{Z}^{2}}
\end{aligned}
$$




$$
\begin{aligned}
\frac{\alpha U}{4 s_{W}^{2}}= & \frac{\Pi_{W W}\left(M_{W}^{2}\right)-\Pi_{W W}(0)}{M_{W}^{2}}-c_{W}^{2} \frac{\Pi_{Z Z}\left(M_{Z}^{2}\right)-\Pi_{Z Z}(0)}{M_{Z}^{2}} \\
& -2 s_{W} c_{W} \frac{\Pi_{Z \gamma}\left(M_{Z}^{2}\right)}{M_{Z}^{2}}-s_{W}^{2} \frac{\Pi_{\gamma \gamma}\left(M_{Z}^{2}\right)}{M_{Z}^{2}} .
\end{aligned}
$$

Here we have followed the definitions given, for example, in [17, which differ slightly from those given originally in [18]. We use the notation $c_{W}$ and $s_{W}$ to refer to the cosine and sine of the weak mixing angle, and $c_{2 W}=c_{W}^{2}-s_{W}^{2}$. All of the self-energy functions $\Pi_{X Y}$ are taken to contain only the new physics contributions (beyond the Standard Model with a Higgs boson), and follow the sign convention of e.g. ref. [17].

In order to completely describe oblique corrections near the $Z$ pole and at zero momentum, it is necessary to introduce three more parameters, as in [19. These can be written in combinations $V, W, Y$, defined as

$$
\begin{aligned}
\alpha Y & =\frac{\Pi_{\gamma \gamma}\left(M_{Z}^{2}\right)}{M_{Z}^{2}}-\hat{\Pi}_{\gamma \gamma}(0), \\
\alpha V & =\Pi_{Z Z}^{\prime}\left(M_{Z}^{2}\right)-\left[\frac{\Pi_{Z Z}\left(M_{Z}^{2}\right)-\Pi_{Z Z}(0)}{M_{Z}^{2}}\right], \\
\alpha W & =\Pi_{W W}^{\prime}\left(M_{Z}^{2}\right)-\left[\frac{\Pi_{W W}\left(M_{W}^{2}\right)-\Pi_{W W}(0)}{M_{W}^{2}}\right],
\end{aligned}
$$

Here $\hat{\Pi}_{\gamma \gamma}\left(p^{2}\right)=\Pi_{\gamma \gamma}\left(p^{2}\right) / p^{2}$, and $\Pi^{\prime}\left(p^{2}\right)=d \Pi / d p^{2}$. The parameter $Y$ we use here is a convenient linear combination of the parameters originally defined in [19]. Note also that ref. [19] used a slightly different definition of $S, T, U$ than used here or in other references.

In terms of the parameters defined above, the observables pertinent to our discussion are expressed as follows:

$$
\begin{aligned}
\frac{M_{W}^{2}}{\left(M_{W}^{2}\right)_{\mathrm{SM}}} & =1-\frac{\alpha S}{2 c_{2 W}}+\frac{c_{W}^{2} \alpha T}{c_{2 W}}+\frac{\alpha U}{4 s_{W}^{2}}-\frac{s_{W}^{2} \alpha Y}{c_{2 W}} . \\
\frac{s_{\mathrm{eff}}^{2}}{\left(s_{\mathrm{eff}}^{2}\right)_{\mathrm{SM}}} & =1+\frac{\alpha S}{4 s_{W}^{2} c_{2 W}}-\frac{c_{W}^{2} \alpha T}{c_{2 W}}+\frac{c_{W}^{2} \alpha Y}{c_{2 W}} . \\
\frac{\Gamma_{l}}{\left(\Gamma_{l}\right)_{\mathrm{SM}}} & =1-d_{W} \alpha S+\left(1+4 s_{W}^{2} c_{W}^{2} d_{W}\right) \alpha T+\alpha V-4 s_{W}^{2} c_{W}^{2} d_{W} \alpha Y,
\end{aligned}
$$

where $X_{\mathrm{SM}}\left(X=M_{W}, s_{\mathrm{eff}}^{2}\right.$ and $\left.\Gamma_{l}\right)$ are the SM values, and $d_{W}=\left(1-4 s_{W}^{2}\right) /\left[\left(1-4 s_{W}^{2}+\right.\right.$ $\left.\left.8 s_{W}^{4}\right) c_{2 W}\right]$. Note that the quantity $W$ does not contribute at all to these particular observables in this parameterization.

\section{Oblique corrections in low-energy supersymmetry}

The preceding analysis applies to a general theory of new physics in which vertex corrections are small. In order to employ these parameter expansions in supersymmetry, we 
need to compute the contributions to the vector boson self-energies. These will be given in terms of kinematic functions $B, H, G, F$, which are defined in the Appendix. They are implicitly functions of an external momentum invariant $s=p^{2}$ (in a signature +--metric). In some special cases, it is often convenient to then expand these kinematic functions in $r=s / M^{2}$, where $M$ is the mass of the heavier particle in the loop, to obtain relatively simple and understandable expressions. The name of a particle stands for its squared mass when appearing as the argument of a kinematic function.

The chargino and neutralino contributions to the electroweak vector boson self-energies are

$$
\begin{aligned}
\Pi_{W W}= & -\frac{g^{2}}{16 \pi^{2}} \sum_{i=1}^{4} \sum_{j=1}^{2}\left[\left(\left|O_{i j}^{L}\right|^{2}+\left|O_{i j}^{R}\right|^{2}\right) H\left(\tilde{\chi}_{i}^{0}, \tilde{\chi}_{j}^{+}\right)+4 \operatorname{Re}\left[O_{i j}^{L} O_{i j}^{R *}\right] M_{\tilde{\chi}_{i}^{0}} M_{\tilde{\chi}_{j}^{+}} B\left(\tilde{\chi}_{i}^{0}, \tilde{\chi}_{j}^{+}\right)\right] \\
\Pi_{Z Z}= & -\frac{g^{2}}{16 \pi^{2} c_{W}^{2}}\left\{\sum_{i, j=1}^{4}\left[\left|O_{i j}^{\prime \prime L}\right|^{2} H\left(\tilde{\chi}_{i}^{0}, \tilde{\chi}_{j}^{0}\right)-2 \operatorname{Re}\left[\left(O_{i j}^{\prime L}\right)^{2}\right] M_{\tilde{\chi}_{i}^{0}} M_{\tilde{\chi}_{j}^{0}} B\left(\tilde{\chi}_{i}^{0}, \tilde{\chi}_{j}^{0}\right)\right]\right. \\
& \left.+\sum_{i, j=1}^{2}\left[\left(\left|O_{i j}^{\prime L}\right|^{2}+\left|O_{i j}^{\prime R}\right|^{2}\right) H\left(\tilde{\chi}_{i}^{+}, \tilde{\chi}_{j}^{+}\right)+4 \operatorname{Re}\left[O_{i j}^{\prime L} O_{i j}^{\prime R *}\right] M_{\tilde{\chi}_{i}^{+}} M_{\tilde{\chi}_{j}^{+}} B\left(\tilde{\chi}_{i}^{+}, \tilde{\chi}_{j}^{+}\right)\right]\right\} \\
\Pi_{Z \gamma}= & \frac{g^{2} s_{W}}{16 \pi^{2} c_{W}} \sum_{i=1}^{2}\left(O_{i i}^{\prime L}+O_{i i}^{\prime R}\right) G\left(\tilde{\chi}_{i}^{+}\right) \\
\Pi_{\gamma \gamma}= & -\frac{g^{2} s_{W}^{2}}{8 \pi^{2}} \sum_{i=1}^{2} G\left(\tilde{\chi}_{i}^{+}\right)
\end{aligned}
$$

The notation for the chargino and neutralino couplings is the same as in 20, 21, and can be described as follows. In the $\left(\widetilde{B}, \widetilde{W}^{0}, \widetilde{H}_{d}^{0}, \widetilde{H}_{u}^{0}\right)$ basis, the neutralino mass matrix is

$$
M_{\tilde{\chi}^{0}}=\left(\begin{array}{cccc}
M_{1} & 0 & -g^{\prime} v_{d} / \sqrt{2} & g^{\prime} v_{u} / \sqrt{2} \\
0 & M_{2} & g v_{d} / \sqrt{2} & -g v_{u} / \sqrt{2} \\
-g^{\prime} v_{d} / \sqrt{2} & g v_{d} / \sqrt{2} & 0 & -\mu \\
g^{\prime} v_{u} / \sqrt{2} & -g v_{u} / \sqrt{2} & -\mu & 0
\end{array}\right),
$$

where $v_{u, d}=\left\langle H_{u, d}^{0}\right\rangle$ and $v_{u} / v_{d}=\tan \beta$, such that $v_{u}^{2}+v_{d}^{2} \simeq(174 \mathrm{GeV})^{2}$. The unitary matrix $N$ diagonalizes $M_{\tilde{\chi}^{0}}$ :

$$
N^{*} M_{\tilde{\chi}^{0}} N^{-1}=\operatorname{diag}\left(M_{\tilde{\chi}_{1}^{0}}, M_{\tilde{\chi}_{2}^{0}}, M_{\tilde{\chi}_{3}^{0}}, M_{\tilde{\chi}_{4}^{0}}\right),
$$

where the mass eigenvalues $M_{\tilde{\chi}_{i}^{0}}$ are all real and positive (see [21] for technique). In the $\left(\widetilde{W}^{ \pm}, \widetilde{H}^{ \pm}\right)$basis, the chargino mass matrix is

$$
M_{\tilde{\chi}^{+}}=\left(\begin{array}{cc}
M_{2} & g v_{u} \\
g v_{d} & \mu
\end{array}\right) .
$$

The unitary matrices $U$ and $V$ diagonalize the above matrix according to

$$
U^{*} M_{\tilde{\chi}^{+}} V^{\dagger}=\left(\begin{array}{cc}
M_{\tilde{\chi}_{1}^{+}} & 0 \\
0 & M_{\tilde{\chi}_{2}^{+}}
\end{array}\right)
$$


where again $M_{\tilde{\chi}_{i}^{+}}$are real and positive. One finds $U$ and $V$ by solving

$$
V M_{\tilde{\chi}^{+}}^{\dagger} M_{\tilde{\chi}^{+}} V^{-1}=U M_{\tilde{\chi}^{+}}^{*} M_{\tilde{\chi}^{+}}^{T} U^{-1}=\left(\begin{array}{cc}
M_{\tilde{\chi}_{1}^{+}}^{2} & 0 \\
0 & M_{\tilde{\chi}_{2}^{+}}^{2}
\end{array}\right) .
$$

The $O_{i j}$-couplings are

$$
\begin{array}{ll}
O_{i j}^{L}=N_{i 2} V_{j 1}^{*}-N_{i 4} V_{j 2}^{*} / \sqrt{2}, & O_{i j}^{R}=N_{i 2}^{*} U_{j 1}+N_{i 3}^{*} U_{j 2} / \sqrt{2}, \\
O_{i j}^{\prime L}=-V_{i 1} V_{j 1}^{*}-\frac{1}{2} V_{i 2} V_{j 2}^{*}+s_{W}^{2} \delta_{i j}, & O_{i j}^{\prime R}=-U_{i 1}^{*} U_{j 1}-\frac{1}{2} U_{i 2}^{*} U_{j 2}+s_{W}^{2} \delta_{i j}, \\
O_{i j}^{\prime \prime L}=\left(-N_{i 3} N_{j 3}^{*}+N_{i 4} N_{j 4}^{*}\right) / 2 . &
\end{array}
$$

In eqs. (3.14) and (3.16), we have assumed that the gaugino couplings to Higgs-higgsino pairs are given by the tree-level supersymmetric relation. We will discuss the merits of this assumption in section 4 .

For completeness, we also compute oblique corrections due to the sfermions and heavy Higgs bosons. As we stated in the introduction, we are assuming that the sfermions and heavy Higgs bosons are decoupled and have no substantive effect on the fits if their masses are above a TeV. The equations below are used to justify that statement.

First, for the sfermions, we assume, as is consistently suggested by experimental constraints and theoretical prejudice, that the first two families have negligible sfermion mixing. For the third family sfermions $\tilde{t}_{i}, \tilde{b}_{i}$, and $\tilde{\tau}_{i}$ with $i=1,2$, the mixing (including possible CP violating phases) is described by

$$
\left(\begin{array}{c}
\tilde{f}_{L} \\
\tilde{f}_{R}
\end{array}\right)=\left(\begin{array}{cc}
c_{\tilde{f}} & -s_{\tilde{f}}^{*} \\
s_{\tilde{f}} & c_{\tilde{f}}^{*}
\end{array}\right)\left(\begin{array}{c}
\tilde{f}_{1} \\
\tilde{f}_{2}
\end{array}\right)
$$

where $\left|c_{\tilde{f}}\right|^{2}+\left|s_{\tilde{f}}\right|^{2}=1$. When there is no CP violation, $c_{\tilde{f}}$ and $s_{\tilde{f}}$ are real and are the sine and cosine of a sfermion mixing angle. Then the sfermion contributions to the self energies of the vector bosons are

$$
\begin{aligned}
\Pi_{W W}= & \frac{g^{2}}{32 \pi^{2}}\left[3 F\left(\tilde{d}_{L}, \tilde{u}_{L}\right)+3 F\left(\tilde{s}_{L}, \tilde{c}_{L}\right)+3 \sum_{i, j=1}^{2}\left|g_{W \tilde{b}_{i} \tilde{t}_{j}^{*}}\right|^{2} F\left(\tilde{b}_{i}, \tilde{t}_{j}\right)\right. \\
& \left.+F\left(\tilde{e}_{L}, \tilde{\nu}_{e}\right)+F\left(\tilde{\mu}_{L}, \tilde{\nu}_{\mu}\right)+\sum_{i=1}^{2}\left|g_{W \tilde{\tau}_{i} \tilde{\nu}_{\tau}^{*}}\right|^{2} F\left(\tilde{\tau}_{i}, \tilde{\nu}_{\tau}\right)\right] \\
\Pi_{Z Z}= & \frac{g^{2}}{16 \pi^{2} c_{W}^{2}} \sum_{f} N_{f} \sum_{i, j}\left|g_{Z \tilde{f}_{i} \tilde{f}_{j}^{*}}\right|^{2} F\left(\tilde{f}_{i}, \tilde{f}_{j}\right) \\
\Pi_{Z \gamma}= & \frac{g^{2} s_{W}}{16 \pi^{2} c_{W}} \sum_{\tilde{f}_{i}} N_{f} Q_{f} g_{Z \tilde{f}_{i} \tilde{f}_{i}^{*}} F\left(\tilde{f}_{i}, \tilde{f}_{i}\right) \\
\Pi_{\gamma \gamma}= & \frac{g^{2} s_{W}^{2}}{16 \pi^{2}} \sum_{\tilde{f}_{i}} N_{f} Q_{f}^{2} F\left(\tilde{f}_{i}, \tilde{f}_{i}\right)
\end{aligned}
$$


Here, $N_{f}=3,1$ and $Q_{f}=+2 / 3,-1 / 3,-1,0$ in the obvious way. In eq. (3.22) the sum on $f$ is over the 12 symbols $\left(d, s, b, u, c, t, e, \mu, \tau, \nu_{e}, \nu_{\mu}, \nu_{\tau}\right)$, and $i, j$ run over 1,2 , except for the sneutrinos. In eqs. (3.23) and (3.24), the sums are over the 18 charged sfermion mass eigenstates. The $Z$ couplings for the first two families and the tau sneutrino are

$$
\begin{aligned}
& g_{Z \tilde{d}_{L} \tilde{d}_{L}^{*}}=-1 / 2+s_{W}^{2} / 3, \quad g_{Z \tilde{u}_{L} \tilde{u}_{L}^{*}}=1 / 2-2 s_{W}^{2} / 3, \quad g_{Z \tilde{e}_{L} \tilde{e}_{L}^{*}}=-1 / 2+s_{W}^{2}, \\
& g_{Z \tilde{\nu} \tilde{\nu}^{*}}=1 / 2, \quad g_{Z \tilde{u}_{R} \tilde{u}_{R}^{*}}=-2 s_{W}^{2} / 3, \quad g_{Z \tilde{d}_{R} \tilde{d}_{R}^{*}}=s_{W}^{2} / 3, \quad g_{Z \tilde{e}_{R} \tilde{e}_{R}^{*}}=s_{W}^{2},
\end{aligned}
$$

and for the third-family sfermions other than the tau sneutrino are

$$
\begin{aligned}
& g_{Z \tilde{f}_{1} \tilde{f}_{1}^{*}}=\left|c_{\tilde{f}}\right|^{2} g_{Z \tilde{f}_{L} \tilde{f}_{L}^{*}}+\left|s_{\tilde{f}}\right|^{2} g_{Z \tilde{f}_{R} \tilde{f}_{R}^{*}}, \quad g_{Z \tilde{f}_{2} \tilde{f}_{2}^{*}}=\left|s_{\tilde{f}}\right|^{2} g_{Z \tilde{f}_{L} \tilde{f}_{L}^{*}}+\left|c_{\tilde{f}}\right|^{2} g_{Z \tilde{f}_{R} \tilde{f}_{R}^{*}}, \\
& g_{Z \tilde{f}_{1} \tilde{f}_{2}^{*}}=\left(g_{Z \tilde{f}_{2} \tilde{f}_{1}^{*}}\right)^{*}=s_{\tilde{f}} c_{\tilde{f}}\left(g_{Z \tilde{f}_{R} \tilde{f}_{R}^{*}}-g_{Z \tilde{f}_{L} \tilde{f}_{L}^{*}}\right) .
\end{aligned}
$$

The $W$ couplings for the third-family sfermions are

$$
\begin{aligned}
& g_{W \tilde{b}_{1} \tilde{t}_{1}^{*}}=c_{\tilde{b}} c_{\tilde{t}}^{*}, \quad g_{W \tilde{b}_{2} \tilde{t}_{2}^{*}}=s_{\tilde{b}}^{*} s_{\tilde{t}}, \quad g_{W \tilde{b}_{1} \tilde{t}_{2}^{*}}=-c_{\tilde{b}} s_{\tilde{t}}, \quad g_{W \tilde{b}_{2} \tilde{t}_{1}^{*}}=-s_{\tilde{b}}^{*} c_{\tilde{t}}^{*}, \\
& g_{W \tilde{\tau}_{1} \tilde{\nu}_{\tau}}=c_{\tilde{\tau}}, \quad g_{W \tilde{\tau}_{2} \tilde{\nu}_{\tau}}=-s_{\tilde{\tau}}^{*} .
\end{aligned}
$$

Finally, we consider the contributions of the Higgs scalar bosons, $h^{0}, H^{0}, A^{0}$, and $H^{ \pm}$. We assume that the Standard Model result already includes contributions from the lightest Higgs scalar. Therefore, to compensate, in the following we subtract a contribution from $h^{0}$ with Standard Model couplings, in other words $\sin ^{2}(\beta-\alpha) \rightarrow 1$. This just converts each term involving $h^{0}$ and $W, Z$ with coefficient $\sin ^{2}(\beta-\alpha)$ into one with coefficient $-\cos ^{2}(\beta-\alpha)$. The results below are therefore the difference between the MSSM and the Standard Model with Higgs mass $m_{h}$ :

$$
\begin{aligned}
\Pi_{W W}= & \frac{g^{2}}{64 \pi^{2}}\left\{F\left(A^{0}, H^{+}\right)+\sin ^{2}(\beta-\alpha) F\left(H^{0}, H^{+}\right)+\cos ^{2}(\beta-\alpha)\left[F\left(h^{0}, H^{+}\right)\right.\right. \\
& \left.\left.+F\left(H^{0}, W\right)-4 M_{W}^{2} B\left(H^{0}, W\right)-F\left(h^{0}, W\right)+4 M_{W}^{2} B\left(h^{0}, W\right)\right]\right\} \\
\Pi_{Z Z}= & \frac{g^{2}}{64 \pi^{2} c_{W}^{2}}\left\{c_{2 W}^{2} F\left(H^{+}, H^{+}\right)+\sin ^{2}(\beta-\alpha) F\left(A^{0}, H^{0}\right)+\cos ^{2}(\beta-\alpha)\left[F\left(h^{0}, A^{0}\right)\right.\right. \\
& \left.\left.+F\left(H^{0}, Z\right)-4 M_{Z}^{2} B\left(H^{0}, Z\right)-F\left(h^{0}, Z\right)+4 M_{Z}^{2} B\left(h^{0}, Z\right)\right]\right\} \\
\Pi_{Z \gamma}= & \frac{g^{2} s_{W} c_{2 W}}{32 \pi^{2} c_{W}} F\left(H^{+}, H^{+}\right) \\
\Pi_{\gamma \gamma}= & \frac{g^{2} s_{W}^{2}}{16 \pi^{2}} F\left(H^{+}, H^{+}\right)
\end{aligned}
$$

\section{Precision fits with light supersymmetric fermions}

We are now in position to compute the effects of light supersymmetric particles and Higgs scalars on precision electroweak observables. In this section we consider the effects of light 
supersymmetric fermions (charginos and neutralinos). We do this in the light gaugino limit first, then the light higgsino limit. These two limits admit nice analytic results, and are interesting to study in their own right. We then consider results for a general mixed higgsino and gaugino scenario.

\section{Light gauginos limit}

In this section we assume that the $\mu$ term is very heavy along with the squarks and sleptons. Thus, we assume all states in the theory decouple except a light SM-like Higgs boson and light gauginos. We then have pure electroweak bino and winos at the low-energy scale. The gluino can also be light, of course, but it has no effect on the electroweak observables. Thus, all results depend on the unknown $h$ mass and the unknown wino mass. Since the pure bino does not couple to $Z, W$, and $\gamma$, it does not contribute to oblique corrections, and so its value with respect to the wino mass is irrelevant to this analysis.

The values of the couplings for the light gaugino limiting case are

$$
O_{21}^{L}=O_{21}^{R}=-O_{11}^{\prime L} / c_{W}^{2}=-O_{11}^{\prime R} / c_{W}^{2}=1,
$$

and all other couplings are either zero or irrelevant. The expressions for the self-energies in this case collapse into a rather convenient form:

$$
\Pi_{W W}=\frac{\Pi_{Z Z}}{c_{W}^{2}}=\frac{\Pi_{Z \gamma}}{s_{W} c_{W}}=\frac{\Pi_{\gamma \gamma}}{s_{W}^{2}}=-\frac{g^{2}}{8 \pi^{2}} G(\tilde{W}) .
$$

The function $G(x)$ is defined in the appendix. The $p^{2}$ argument of $G(x)$, and of other loop functions that we will define later, is not explicitly written for simplicity of notation.

We note immediately that $S=T=0$ for this case. Thus, an $S-T$ parameter analysis cannot capture the effects of winos on precision electroweak observables. The non-zero contributions to the precision electroweak observables come from the $U, Y$ and $V$ parameters. These parameters can be computed straightforwardly given their definitions and eq. (4.2). We use these to obtain a convenient expansion of the observables in powers of $r_{\tilde{W}}=M_{Z}^{2} / M_{2}^{2}$ :

$$
\begin{aligned}
\Delta M_{W}(\mathrm{GeV}) & =0.00954 r_{\tilde{W}}+0.00157 r_{\tilde{W}}^{2}+0.00030 r_{\tilde{W}}^{3}, \\
\Delta s_{\text {eff }}^{2} & =-0.0000549 r_{\tilde{W}}-0.0000059 r_{\tilde{W}}^{2}-0.0000009 r_{\tilde{W}}^{3}, \\
\Delta \Gamma_{l}(\mathrm{MeV}) & =-0.0435 r_{\tilde{W}}-0.0096 r_{\tilde{W}}^{2}-0.0022 r_{\tilde{W}}^{3} .
\end{aligned}
$$

We have found numerically that this expansion is quite accurate even when $r_{\tilde{W}}$ is near one, provided that $|\mu|$ is large.

Note that in the above equations $\Delta M_{W}>0, \Delta s_{\text {eff }}^{2}<0$ and $\Delta \Gamma_{l}<0$ for the wino corrections. Comparing the SM predictions in Table 1 with the experimental data in 


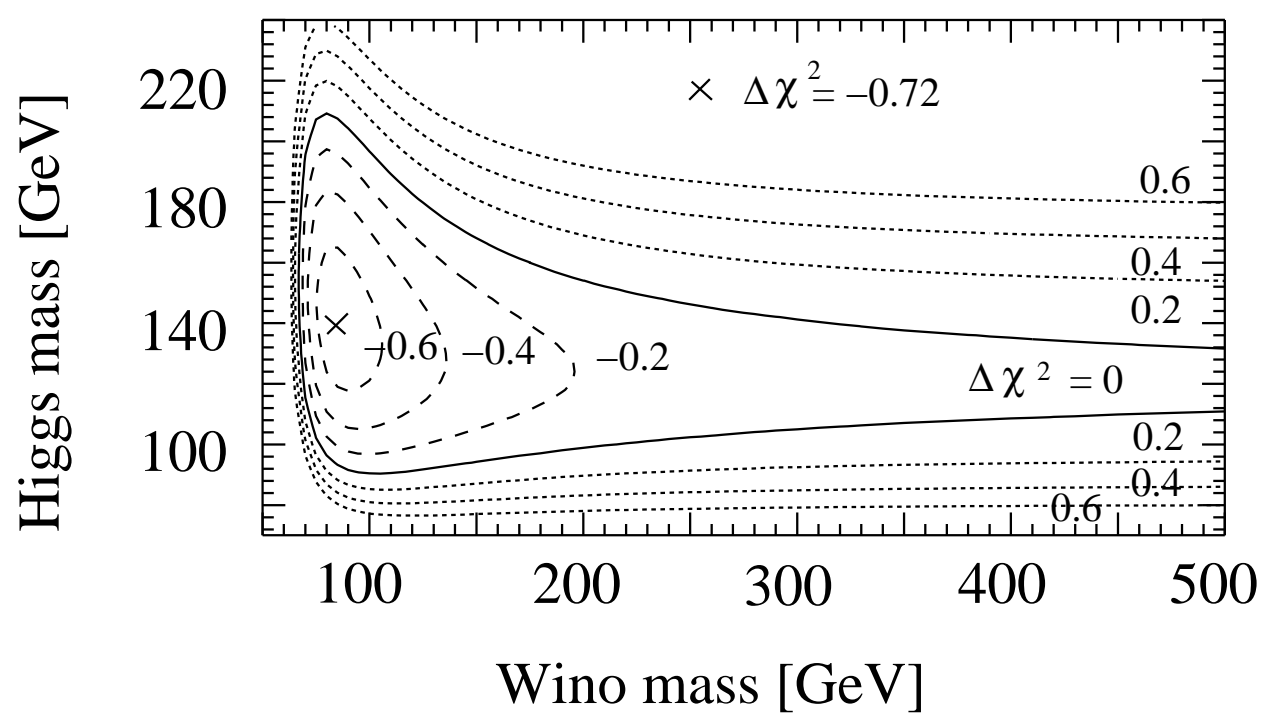

Figure 2: Contours of $\Delta \chi^{2}=\chi^{2}-\chi_{S M \text {, min }}^{2}$ as a function of Higgs mass $M_{h}$ and the wino mass $M_{2}$. The region inside (outside) of the solid line produces a better (worse) fit than the best fit point of the Standard Model, which corresponds to the limit of superpartner masses decoupling to infinity and $M_{h}=120 \mathrm{GeV}$. The best fit point, not taking into account direct searches for the charged wino, is indicated by the point marked $\times$ at $M_{h}=141 \mathrm{GeV}$, $M_{2}=86 \mathrm{GeV}$. This figure was made using $\alpha_{s}\left(M_{Z}\right)=0.1187$.

eqs. (2.1)-(2.3), the light winos improve $M_{W}$ and $\Gamma_{l}$ predictions. To see how light winos can affect these observables, we consider "0.5- $\sigma$ sensitive wino mass" which changes the SM predictions of observables by $0.5-\sigma$. The current experimental uncertainties for observables are $\delta M_{W}=0.034 \mathrm{GeV}, \delta s_{\text {eff }}^{2}=0.00017$ and $\delta \Gamma_{l}=0.086 \mathrm{MeV}$ at $1-\sigma$ level, and hence we can calculate " $0.5-\sigma$ sensitive wino mass" using the above expansions. They are $M_{2}=77$ $\mathrm{GeV}$ from $\Delta M_{W}=\delta M_{W} / 2, M_{2}=79 \mathrm{GeV}$ from $\Delta s_{\text {eff }}^{2}=\delta s_{\text {eff }}^{2} / 2$ and $M_{2}=101 \mathrm{GeV}$ from $\Delta \Gamma_{l}=\delta \Gamma_{l} / 2$. Therefore winos with $M_{2} \sim 100 \mathrm{GeV}$ can affect the electroweak fit. Note that $\Gamma_{l}$ is the most sensitive to the light winos and it is improved.

In fig. 2, we show the total $\Delta \chi^{2}=\chi^{2}-\chi_{S M, \text { min }}^{2}$ for the electroweak fit as a function of Higgs mass $M_{h}$ and wino mass $M_{2}$. As one can see, the $\chi^{2}$ improves as $M_{2}$ gets smaller (up to about $90 \mathrm{GeV}$ ) in the range of $115 \mathrm{GeV}<M_{h}<170 \mathrm{GeV}$. The minimum of the $\chi^{2}$ is about 0.95 at $M_{h} \simeq 140 \mathrm{GeV}$ and $M_{2} \simeq 85 \mathrm{GeV}$. Note that the minimum of the $\chi^{2}$ in the $\mathrm{SM}$ is about $\chi_{S M, \min }^{2}=1.7$ at $M_{h}=120 \mathrm{GeV}$ (see fig. 11 and Table 1). Note also that the current wino mass limit is about $90 \mathrm{GeV}$ when all squarks and higgsinos are heavy [22].

\section{Light higgsinos limit}

Next, we consider a different limit: all gaugino masses are large, but $\mu$ is small. In this case, low-energy charginos and neutralinos are pure higgsinos. All precision electroweak 
results are functions of the light SM-like Higgs mass and the higgsino mass $\mu$.

The light higgsino case corresponds to $O_{11}^{L}=O_{11}^{R}=-1 / 2$, and $O_{21}^{L}=O_{21}^{R}=i / 2$, $O_{11}^{\prime L}=O_{11}^{\prime R}=s_{W}^{2}-1 / 2, O_{11}^{\prime \prime L}=O_{22}^{\prime \prime L}=0$, and $O_{12}^{\prime \prime L}=-O_{21}^{\prime \prime L}=i / 2$, and all others are irrelevant. The vector boson self energies reduce to

$$
\Pi_{W W}=\frac{c_{W}^{2}}{c_{W}^{4}+s_{W}^{4}} \Pi_{Z Z}=\frac{c_{W}}{s_{W}\left(c_{W}^{2}-s_{W}^{2}\right)} \Pi_{Z \gamma}=\frac{1}{2 s_{W}^{2}} \Pi_{\gamma \gamma}=-\frac{g^{2}}{16 \pi^{2}} G(\tilde{H})
$$

Similar to the pure gaugino limit, $S=T=0$ in this limit, and $S-T$ analysis alone cannot capture the effects of light higgsinos on precision electroweak observables.

We can also expand the pure higgsino limit analytically as a power series in $r_{\tilde{H}}=m_{Z}^{2} / \mu^{2}$ :

$$
\begin{aligned}
\Delta M_{W}(\mathrm{GeV}) & =0.00620 r_{\tilde{H}}+0.00094 r_{\tilde{H}}^{2}+0.00017 r_{\tilde{H}}^{3} \\
\Delta s_{\text {eff }}^{2} & =-0.0000549 r_{\tilde{H}}-0.0000059 r_{\tilde{H}}^{2}-0.0000009 r_{\tilde{H}}^{3} \\
\Delta \Gamma_{l}(\mathrm{MeV}) & =-0.0225 r_{\tilde{H}}-0.0051 r_{\tilde{H}}^{2}-0.0012 r_{\tilde{H}}^{3}
\end{aligned}
$$

The higgsinos also improve $M_{W}$ and $\Gamma_{l}$ compared to the SM predictions. Again, we can calculate "0.5- $\sigma$ sensitive higgsino mass": $\mu=65 \mathrm{GeV}$ from $\Delta M_{W}=\delta M_{W} / 2, \mu=79 \mathrm{GeV}$ from $\Delta s_{\text {eff }}^{2}=\delta s_{\text {eff }}^{2} / 2$ and $\mu=78 \mathrm{GeV}$ from $\Delta \Gamma_{l}=\delta \Gamma_{l} / 2$. (Recall from above that $\delta \mathcal{O}_{i}$ is the $1 \sigma$ experimental error for observable $\mathcal{O}_{i}$.) The total $\Delta \chi^{2}$ is shown in fig. 3 as a function of $\mu$ and Higgs mass $M_{h}$. The effect is smaller than what we found in the pure wino case, but the light higgsinos (with $\mu>80 \mathrm{GeV}$ ) also improve the total $\chi^{2}$ compared to the SM fit.

\section{Mixed gauginos and higgsinos}

Here we consider the more general case for light charginos and neutralinos. When the $\mu$ term is near in mass to the wino mass term $M_{2}$, both the higgsino and wino sectors contribute substantially to the oblique corrections. In this case the general mixing matrix angles for the charginos and neutralinos vary over large ranges. Unfortunately, there are no simple analytic equations that capture all the effects succinctly. All results for the mixed case will be numerical results taking into account proper diagonalizations of the mass matrices.

Unlike the pure gaugino and the pure higgsino cases, the mixed-case results strongly depend on $\tan \beta$. This is easy to understand since the gaugino/higgsino mass mixing insertions are $\tan \beta$ dependent and not small. Thus, $\tan \beta$ is a crucial parameter to keep track of. Another parameter that we should keep track of is the ratio of the higgsino parameter to the wino parameter, $\mu / M_{2}$. We will assume that this mixing parameter can be anything along the real line (no complex, $\mathrm{CP}$ violating phases). 


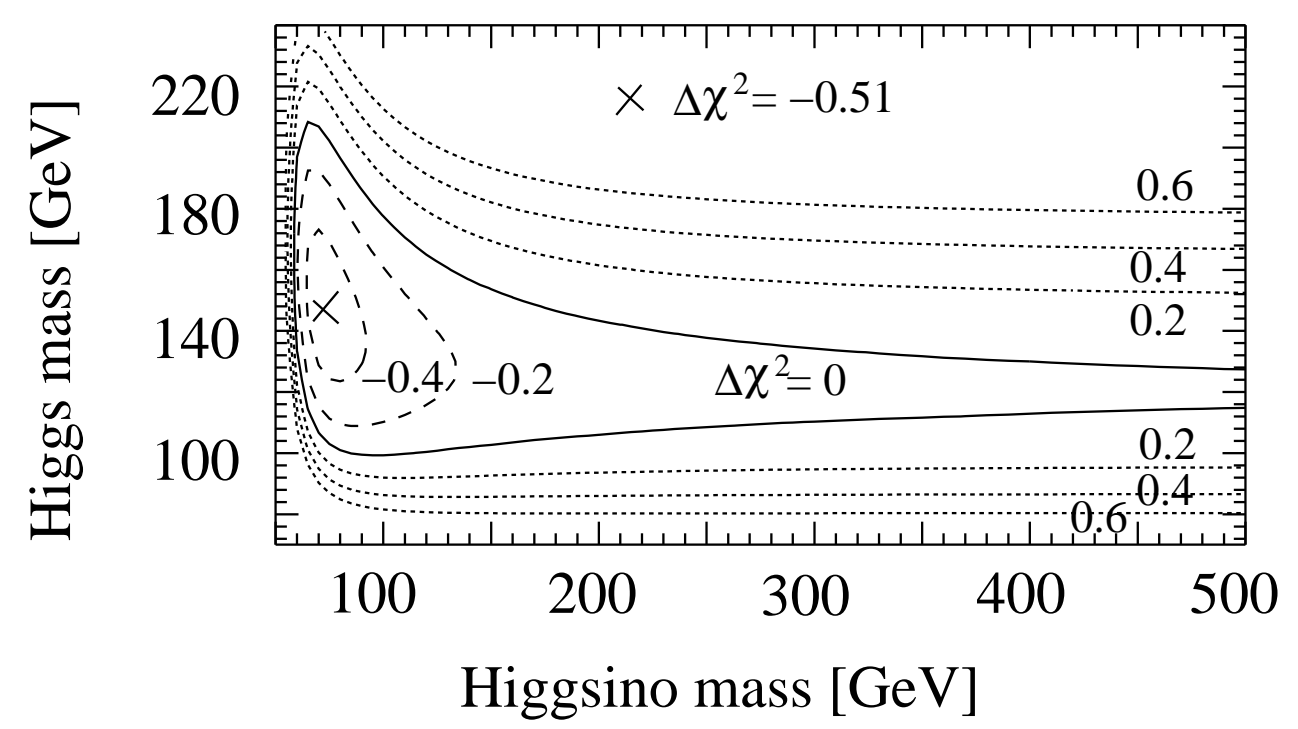

Figure 3: Contours of $\Delta \chi^{2}=\chi^{2}-\chi_{S M \text {,min }}^{2}$ as a function of Higgs mass $M_{h}$ and the higgsino mass. The region inside (outside) of the solid line produces a better (worse) fit than the best fit point of the Standard Model, which corresponds to the limit of all superpartners decoupling to infinite mass and $M_{h}=120 \mathrm{GeV}$. The best fit point, not taking into account direct searches for the charged higgsino, is indicated by the point marked $\times$ at Higgs mass of $147 \mathrm{GeV}$ and higgsino mass of $73 \mathrm{GeV}$. This figure was made using $\alpha_{s}\left(M_{Z}\right)=0.1187$.

We demonstrate the effect of mixing by plotting contours of shifts in observables in the

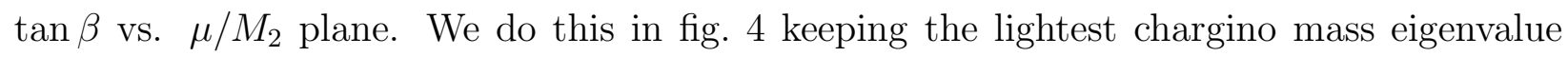
fixed at $M_{\tilde{\chi}_{1}^{+}}=120 \mathrm{GeV}$. We also assume that $M_{1} \simeq 3 M_{2}$ according to anomaly mediated supersymmetry [23], although we have checked that the results depend very mildly on this assumption. Choosing $M_{1} \simeq M_{2}$ or $M_{1} \simeq M_{2} / 2$, for example, generates very similar figures.

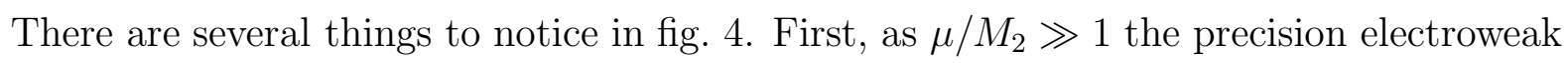
analysis asymptotes to that of light wino superpartners. As $\mu / M_{2} \ll 1$ the precision electroweak analysis asymptotes to that of light higgsinos. In both cases, the $\tan \beta$ dependence disappears and the variation of the corrections to the observables disappears when there

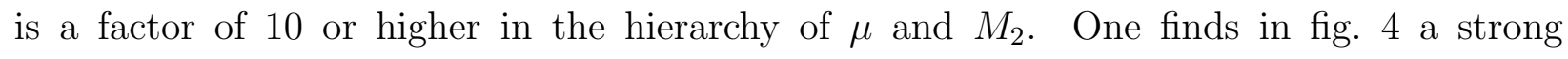
$\tan \beta$ dependence when $\mu / M_{2} \sim 1$, which can induce a correction of either sign for $\Delta M_{W}$, depending on the value of $\tan \beta$, and only positive (negative) corrections to $s_{\text {eff }}^{2}\left(\Gamma_{l}\right)$. The variability in the corrections is large in that region.

Having established that the observables change significantly when $\mu \simeq M_{2}$, we now wish to determine the effect these variations have on $\Delta \chi^{2}$. To this end we have plotted in fig. 5 contours of $\Delta \chi^{2}$ in the plane of $M_{h}$ versus $\mu / M_{2}$. We have fixed $M_{\tilde{\chi}_{1}^{+}}=120 \mathrm{GeV}$, and $\tan \beta=2$ in the top graph and $\tan \beta=50$ in the bottom graph. 

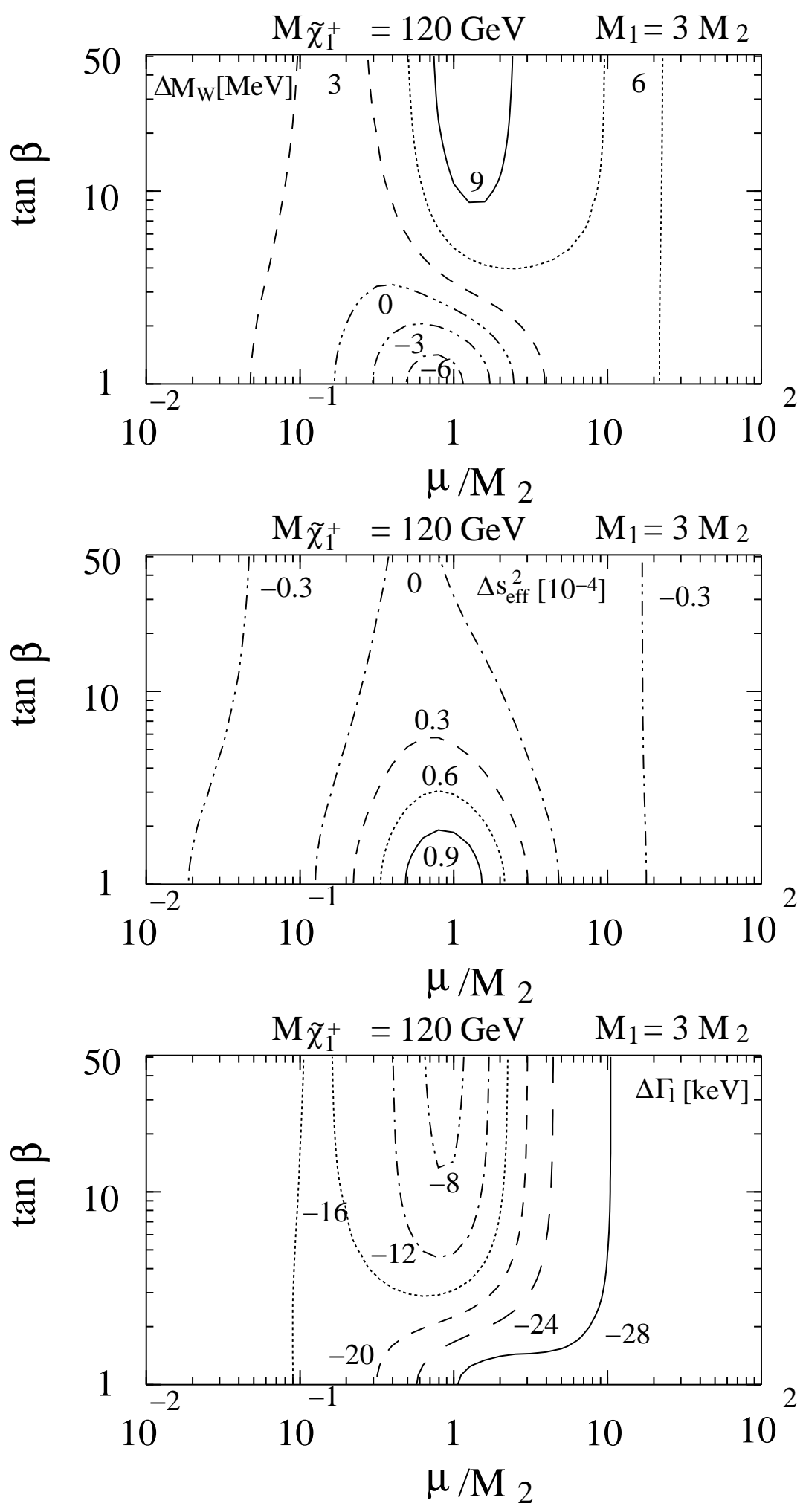

Figure 4: Contours of shifts in electroweak observables in the $\tan \beta$ vs. $\mu / M_{2}$ plane, with the lightest chargino mass eigenstate fixed at $120 \mathrm{GeV}$ and $M_{1}=3 M_{2}$. 

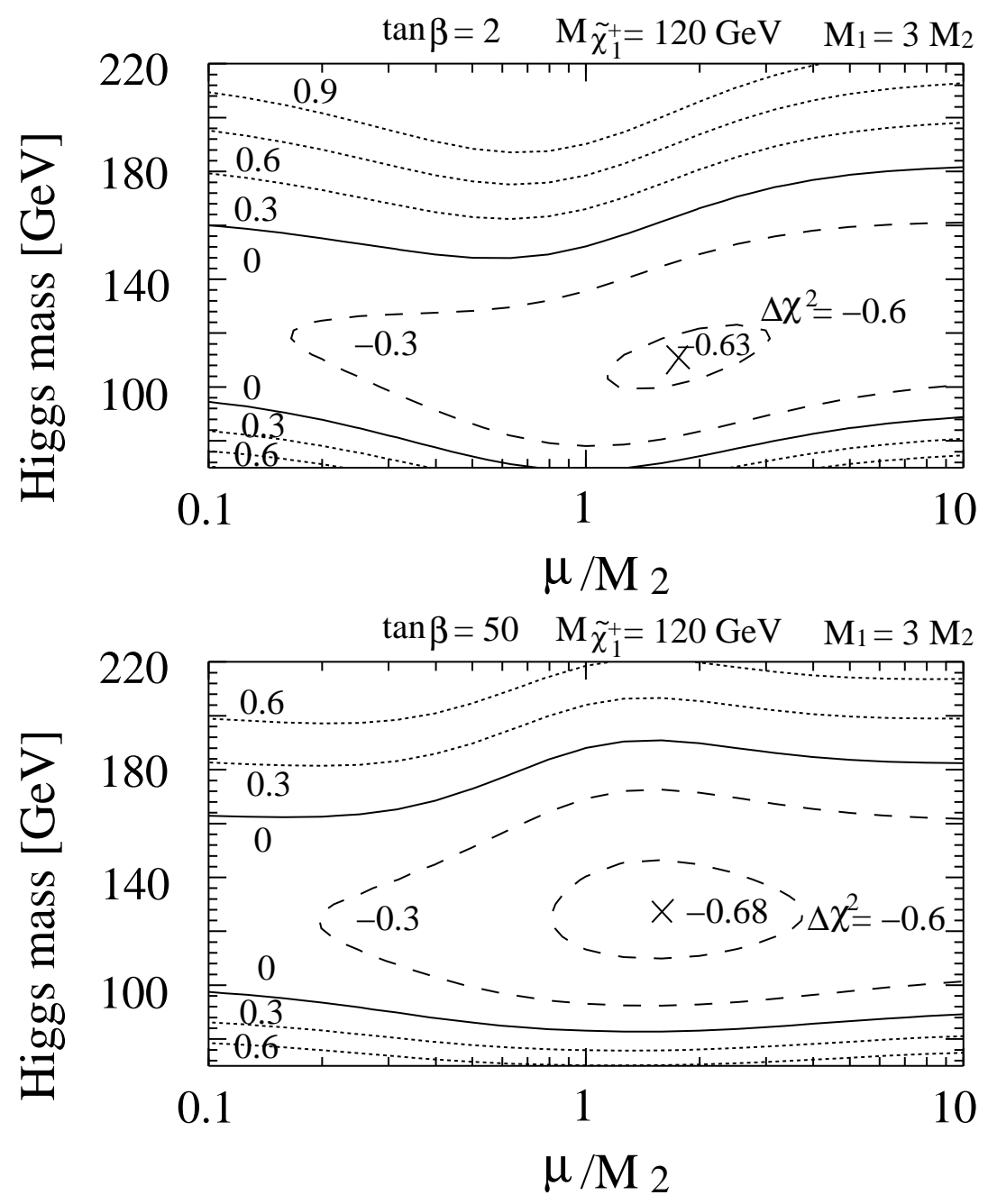

Figure 5: $\Delta \chi^{2}$ contours with $\tan \beta=2,50$ and $M_{\tilde{\chi}_{1}^{+}}=120 \mathrm{GeV}$ fixed. We have also chosen $M_{1}=3 M_{2}$, although the contours depend very mildly on this assumption. The results illustrate the general finding that a mixed scenario of light gauginos and higgsinos lead to a better fit to the precision electroweak data. This figure was made using $\alpha_{s}\left(M_{Z}\right)=0.1187$. 
On the far left of the figure we have the result of the pure higgsino case, where the $\Delta \chi^{2}$ changes only when the Higgs boson mass changes. The contour lines become parallel to the $x$-axis. On the far right of figure we find the result asymptoting toward the pure gaugino case. Again, $\Delta \chi^{2}$ changes only due to the Higgs boson mass, and the lines again level horizontally out there. In the center of the contours of fig. 5 the mixing angles are varying significantly. The variation is causing the changes in the observables that we witnessed in fig. 4, and subsequently affects $\Delta \chi^{2}$. As we see, the mixing effect reduces $\Delta \chi^{2}$.

Reduction of $\Delta \chi^{2}$ in the region of parameter space where gauginos and higgsinos are light and heavily mixed is a general result in split supersymmetry. We can understand this result from the graphs. Let us draw our attention to a segment of the graphs at $\mu / M_{2} \sim 1$. At high $\tan \beta$ the corrections to $s_{\text {eff }}^{2}$ and $\Gamma_{l}$ are becoming small, whereas the correction to $M_{W}$ is increasing. The increasing contribution to $M_{W}$ makes the theory prediction come closer to the experimental prediction, thus reducing the $\Delta \chi^{2}$. At low $\tan \beta$ the contribution to $M_{W}$ is negative. Although this goes in the wrong direction, the magnitude is somewhat smaller than in the large $\tan \beta$ case, and more importantly, the contributions to $s_{\text {eff }}^{2}$ are increasingly positive, which goes in the right direction, and the contributions to $\Gamma_{l}$ are increasingly negative, which also goes in the right direction. Overall, the $\chi^{2}$ improves. This result is $\operatorname{true}$ for all $\tan \beta$ although we have only shown it graphically for $\tan \beta=2$ and $\tan \beta=50$. The improved $\Delta \chi^{2}$ results also hold similarly for $\mu / M_{2} \simeq-1$. Therefore, mixed higgsinos and gauginos near the direct experimental limit is the split supersymmetry spectrum most compatible with the precision electroweak data.

Let us say a few words about dark matter [2, 4, 5] in relation to our precision electroweak analysis. If we assume that R-parity is conserved, the lightest supersymmetric partner (LSP) will be stable. In the case of a pure wino LSP, the dark matter thermal relic abundance is negligible unless the mass is about 2.3 TeV. Pure higgsino LSP has negligible relic abundance unless its mass is about $1.2 \mathrm{TeV}$. Higher masses mean overclosure, i.e., cosmological problems. However, these conclusions are applicable for thermal relic abundance calculations. Non-thermal sources, such as gravitino or moduli decay in the early universe can transform what looked to be a negligibly abundant LSP into a good dark matter candidate. Thus, the light winos, light higgsinos and light mixed states are probably not good thermal dark matter candidates, but could be good dark matter candidates when all non-thermal sources are taken into account. If $M_{1}<M_{2}$ and the LSP has significant bino fraction, one expects either the LSP to annihilate efficiently through a Higgs boson pole or $\mu$ should be somewhat near $M_{1}$ to mix with the bino for acceptable dark matter (see Pierce in [4]). This is good for dark matter and good for precision electroweak fits. 


\section{Indirect effects from heavy scalars}

In the above analysis we have assumed that the neutralino and chargino matrices used to obtain the mass eigenvalues and mixing angles are valid. However, the $g$ and $g^{\prime}$ that are in those matrices are gauge couplings because of supersymmetry invariance. In broken supersymmetry those couplings deviate from the gauge couplings, which has been emphasized within the context of split supersymmetry [1, [].

Unfortunately, these "gauge-ino couplings" cannot be directly measured by experiment since the scalar masses are likely to not be accessible. However, they can have a subtle effect on the precision electroweak observables. To demonstrate, we introduce the following couplings

$$
\begin{aligned}
a_{u} & =\tilde{g}_{u} /(g \sin \beta), & a_{d} & =\tilde{g}_{d} /(g \cos \beta) \\
a_{u}^{\prime} & =\tilde{g}_{u}^{\prime} /\left(g^{\prime} \sin \beta\right), & a_{d}^{\prime} & =\tilde{g}_{d}^{\prime} /\left(g^{\prime} \cos \beta\right)
\end{aligned}
$$

where $\tilde{g}_{u, d}$ and $\tilde{g}_{u, d}^{\prime}$ are defined in [3]. The $a$-variables are defined such that the usual values taken in the MSSM are $a_{u}=a_{d}=a_{u}^{\prime}=a_{d}^{\prime}=1$.

The neutralino and chargino mass matrices at the weak scale in this parameterization are

$$
\begin{gathered}
M_{\tilde{\chi}^{0}}=\left(\begin{array}{cccc}
M_{1} & 0 & -a_{d}^{\prime} g^{\prime} v_{d} / \sqrt{2} & a_{u}^{\prime} g^{\prime} v_{u} / \sqrt{2} \\
0 & M_{2} & a_{d} g v_{d} / \sqrt{2} & -a_{u} g v_{u} / \sqrt{2} \\
-a_{d}^{\prime} g^{\prime} v_{d} / \sqrt{2} & a_{d} g v_{d} / \sqrt{2} & 0 & -\mu \\
a_{u}^{\prime} g^{\prime} v_{u} / \sqrt{2} & -a_{u} g v_{u} / \sqrt{2} & -\mu & 0
\end{array}\right), \\
M_{\tilde{\chi}^{+}}=\left(\begin{array}{cc}
M_{2} & a_{u} g v_{u} \\
a_{d} g v_{d} & \mu
\end{array}\right)
\end{gathered}
$$

We have computed the numerical values of the $a$-variables under different assumptions for $\tan \beta$ and the scale of the scalar superpartners. (We have reproduced fig. 5 of 3 and agree with their results.) We then compute the corrections to the precision electroweak observables for various values of the scalar sector mass, which for simplicity we assume is a common scale $M_{s}$. For PeV-scale sfermion masses none of the $a$-variables deviate from 1 by more than $10 \%$ for any value of $\tan \beta$. If the scalar masses are near the GUT scale, the effects can be more sizable and deviations from $a_{i}=1$ can approach $30 \%$ at very high $\tan \beta \sim 50$, but are less significant for lower $\tan \beta$.

We note that the effects of various $M_{s}$ values can cause the magnitude of the oblique correction to change by as much as $30 \%$ at special points such as when $M_{2} \simeq|\mu| \simeq 100 \mathrm{GeV}$ 
and $M_{s} \simeq 10^{16} \mathrm{GeV}$. This is especially true for $s_{\text {eff }}^{2}$, which is very sensitive to the chargino and neutralino mixing angles. However, when $\mu$ deviates from $M_{2}$ one finds the effects on precision electroweak corrections to be much smaller. In all cases, the corrections to the oblique corrections are not discernible by current experiment. This is why we ignored these $a_{u, d}^{\prime}$ and $a_{u, d}$ parameters for much of our analysis. In the future, these small deviations might be discernible at dedicated next-generation $Z$ factories $[24]$.

\section{Conclusion}

We have found above that the precision electroweak corrections from light charginos and neutralinos generally improve the overall $\chi^{2}$ fit to the data. This is true in the pure gaugino limit and in the pure higgsino limit. We emphasize that in both of these cases we have merely added one new parameter to the theory and the fit gets better. It did not have to go this direction. In the mixed higgsino and gaugino case, there are more free parameters introduced, and it turns out that the fit to the data gets even better. The case of higgsinos and gauginos both near the direct experimental limit $(\sim 100 \mathrm{GeV})$ is the split supersymmetry spectrum most compatible with the data.

These conclusions are made within the full supersymmetric framework, where we have assumed the scalar superpartner masses are too heavy to have any noticeable effect on the precision electroweak observables. Even for scalar superpartners decoupled up to the grand unification scale, the light Higgs mass is still less than about $170 \mathrm{GeV}$ [3]. Our best fit results are all compatible with this low range of Higgs mass, and the global fits for the various cases we discussed have global minima with light Higgs boson mass. The global fit to the data approaches that of the SM fit when the gaugino and higgsino masses are dialed to larger values. In that case, the global minimum of the $\chi^{2}$ fit remains in the low Higgs mass region, but the $\chi^{2}$ value at that SM minimum is increased somewhat compared to the light gauginos/higgsino case.

Throughout we have assumed that the squarks, sleptons and heavy Higgs bosons have no effect on precision electroweak analysis. Our computations demonstrate that we expect less than a $10^{-4}$ effect on all relative corrections of the observables, $\Delta \mathcal{O}_{i} / \mathcal{O}_{i}$, if the scalar masses are above $1 \mathrm{TeV}$. In split supersymmetry, we expect the scalar masses to be significantly beyond the TeV scale, justifying our neglect of the scalar masses. As an aside, we have found that lowering the sfermion masses usually does not improve the quality of fit compared to the decoupling limit, for fixed values of other quantities. Although not statistically significant, 
the precision electroweak data may have a mild preference for decoupled scalars and light gauginos/higgsinos over any other form of supersymmetry breaking patterns.

In short, the precision electroweak data is compatible with split supersymmetry spectrum for all values of gaugino and higgsino masses above direct collider limits. Near the direct limits, the overall fit improves by nearly a full unit in $\Delta \chi^{2}$. A priori, fitting to precision electroweak observables did not have to be favorable to split supersymmetry, and could have been incompatible for light gauginos and/or higgsinos. As it is, the improved fits are mildly encouraging for the scenario.

Note Added: Following the appearance of the present work, a recent interesting paper 25] suggests that high-energy LEP2 data can contribute substantively to precision electroweak analysis of light superpartners. Although we have not independently confirmed this analysis, we want to bring it to the reader's attention. If correct, the conclusion from doing a precision electroweak analysis with that super-set of observables would eliminate the mild preference for light charginos and neutralinos. As noted in ref. [25], the analysis might change again after all $e^{+} e^{-} \rightarrow e^{+} e^{-}$data above the $Z$ pole becomes available.

\section{Appendix: Useful functions}

The kinematic loop-integral functions needed above are given in terms of

$$
B(x, y)=-\int_{0}^{1} d t \overline{\ln }[t x+(1-t) y-t(1-t) s-i \epsilon]
$$

where $s=p^{2}$ is the external momentum invariant in a (+---) metric, and

$$
\overline{\ln } X \equiv \ln \left(X / Q^{2}\right)
$$

where $Q$ is the renormalization scale. In the text, the arguments $(x, y)$ are particle names and should be interpreted to substitute $x \rightarrow m_{x}^{2}$ and $y \rightarrow m_{y}^{2}$ into the equations.

The other functions used in the text are defined in terms of $B(x, y)$ :

$$
\begin{aligned}
H(x, y)= & {\left[2 s-x-y-(x-y)^{2} / s\right] B(x, y) / 3 } \\
& +[2 x \overline{\ln } x+2 y \overline{\ln } y-2 s / 3+(y-x)(x \overline{\ln } x-x-y \overline{\ln } y+y) / s] / 3 \\
F(x, y)= & H(x, y)+(x+y-s) B(x, y) \\
G(x)= & H(x, x)+2 x B(x, x) .
\end{aligned}
$$

These can be expanded in powers of $s$ according to:

$$
B(x, y)=b_{0}(x, y)+s b_{1}(x, y)+s^{2} b_{2}(x, y)+\ldots
$$




$$
\begin{aligned}
H(x, y) & =h_{0}(x, y)+s h_{1}(x, y)+s^{2} h_{2}(x, y)+\ldots \\
F(x, y) & =f_{0}(x, y)+s f_{1}(x, y)+s^{2} f_{2}(x, y)+\ldots \\
G(x) & =s g_{1}(x)+s^{2} g_{2}(x)+\ldots
\end{aligned}
$$

with coefficients that follow from

$$
\begin{aligned}
b_{0}(x, y)= & {[x-x \overline{\ln } x-y+y \overline{\ln } y] /(x-y), } \\
b_{1}(x, y)= & {\left[2 x y \ln (y / x)+x^{2}-y^{2}\right] / 2(x-y)^{3}, } \\
b_{2}(x, y)= & {\left[6 x y(x+y) \ln (y / x)+x^{3}-y^{3}+9 x^{2} y-9 x y^{2}\right] / 6(x-y)^{5}, } \\
b_{3}(x, y)= & {\left[12 x y\left(x^{2}+3 x y+y^{2}\right) \ln (y / x)+x^{4}-y^{4}+28 x^{3} y-28 x y^{3}\right] / 12(x-y)^{7}, } \\
b_{4}(x, y)= & {\left[60 x y\left(x^{3}+6 x^{2} y+6 x y^{2}+y^{3}\right) \ln (y / x)+3 x^{5}-3 y^{5}+175 x y\left(x^{3}-y^{3}\right)\right.} \\
& \left.+300 x^{2} y^{2}(x-y)\right] / 60(x-y)^{9}
\end{aligned}
$$

and

$$
\begin{array}{ll}
b_{0}(x, x)=-\overline{\ln }(x), & b_{1}(x, x)=1 / 6 x, \quad b_{2}(x, x)=1 / 60 x^{2}, \\
b_{3}(x, x)=1 / 420 x^{3}, & b_{4}(x, x)=1 / 2520 x^{4} .
\end{array}
$$

The expansions converge provided that $\sqrt{s}<\sqrt{x}+\sqrt{y}$ (in other words, below the threshold branch cut).

\section{Acknowledgements}

The work of SPM was supported by the National Science Foundation under Grant No. PHY0140129. KT acknowledges support from the National Science Foundation. The work of JDW was supported in part by the Department of Energy and the Michigan Center for Theoretical Physics. We thank G. Kane and T. Wang for discussions.

\section{References}

[1] N. Arkani-Hamed and S. Dimopoulos, hep-th/0405159.

[2] J. D. Wells, hep-ph/0306127; J. D. Wells, hep-ph/0411041.

[3] G. F. Giudice and A. Romanino, Nucl. Phys. B 699, 65 (2004) hep-ph/0406088. 
[4] A. Arvanitaki, C. Davis, P. W. Graham and J. G. Wacker, hep-ph/0406034 A. Pierce, Phys. Rev. D 70, 075006 (2004) hep-ph/0406144; W. Kilian, T. Plehn, P. Richardson and E. Schmidt, hep-ph/0408088; J. L. Hewett, B. Lillie, M. Masip and T. G. Rizzo, JHEP 0409, 070 (2004) hep-ph/0408248; N. Arkani-Hamed, S. Dimopoulos, G. F. Giudice and A. Romanino, hep-ph/0409232.

[5] M. Beccaria, F. M. Renard and C. Verzegnassi, hep-ph/0412257. M. A. Diaz and P. F. Perez, hep-ph/0412066, A. Masiero, S. Profumo and P. Ullio, hep-ph/0412058, L. Anchordoqui, H. Goldberg and C. Nunez, hep-ph/0408284; B. Mukhopadhyaya and S. SenGupta, hep-th/0407225 V. Barger, C. W. Chiang, J. Jiang and T. Li, Nucl. Phys. B 705, 71 (2005) hep-ph/0410252 ; E. J. Chun and S. C. Park, hep-ph/0410242, U. Sarkar, hep-ph/0410104 K. Cheung and W. Y. Keung, hep-ph/0408335 S. K. Gupta, P. Konar and B. Mukhopadhyaya, hep-ph/0408296 S. h. Zhu, Phys. Lett. B 604, 207 (2004) hep-ph/0407072; R. Allahverdi, A. Jokinen and A. Mazumdar, hep-ph/0410169. P. C. Schuster, hep-ph/0412263.

[6] For various representative views on how string/M-theory/Landscape may impact the scale of supersymmetry breaking, see M. R. Douglas, hep-th/0405279 M. Dine, E. Gorbatov and S. Thomas, hep-th/0407043; I. Antoniadis and S. Dimopoulos, hep-th/0411032; C. Kokorelis, hep-th/0406258, B. Kors and P. Nath, hep-th/0411201; K. R. Dienes, E. Dudas and T. Gherghetta, hep-th/0412185.

[7] J. A. Grifols and J. Sola, Nucl. Phys. B 253, 47 (1985); R. Barbieri, M. Frigeni, F. Giuliani and H. E. Haber, Nucl. Phys. B 341, 309 (1990); J. R. Ellis, S. Kelley and D. V. Nanopoulos, Phys. Lett. B 249, 441 (1990); M. Drees and K. Hagiwara, Phys. Rev. D 42, 1709 (1990); M. Drees, K. Hagiwara and A. Yamada, Phys. Rev. D 45, 1725 (1992); P. H. Chankowski et al., Nucl. Phys. B 417, 101 (1994); P. Chankowski, S. Pokorski and J. Rosiek, Nucl. Phys. B 423, 437 (1994) hep-ph/9303309; G. L. Kane, R. G. Stuart and J. D. Wells, Phys. Lett. B 354, 350 (1995) hep-ph/9505207; D. M. Pierce, J. A. Bagger, K. T. Matchev and R. j. Zhang, Nucl. Phys. B 491, 3 (1997) hep-ph/9606211; W. de Boer, A. Dabelstein, W. Hollik, W. Mosle and U. Schwickerath, Z. Phys. C 75, 627 (1997) hep-ph/9607286; J. Erler and D. M. Pierce, Nucl. Phys. B 526, 53 (1998) hep-ph/9801238.

[8] G. C. Cho and K. Hagiwara, Nucl. Phys. B 574, 623 (2000) hep-ph/9912260; G. Altarelli, F. Caravaglios, G. F. Giudice, P. Gambino and G. Ridolfi, JHEP 0106, 018 (2001) hep-ph/0106029 ; J. R. Ellis, S. Heinemeyer, K. A. Olive and G. Weiglein, hep-ph/0411216, S. Heinemeyer, W. Hollik and G. Weiglein, hep-ph/0412214. 
[9] M. E. Peskin and J. D. Wells, Phys. Rev. D 64, 093003 (2001) hep-ph/0101342.

[10] LEP Collaborations et al., "A combination of preliminary electroweak measurements and constraints on the standard model," hep-ex/0312023, hep-ex/0412015

[11] P. B. Renton, hep-ph/0410177

[12] M. Awramik, M. Czakon, A. Freitas and G. Weiglein, Phys. Rev. Lett. 93, 201805 (2004) hep-ph/0407317.

[13] M. Awramik, M. Czakon, A. Freitas and G. Weiglein, Phys. Rev. D 69, 053006 (2004) hep-ph/0311148.

[14] A. Ferroglia, G. Ossola, M. Passera and A. Sirlin, Phys. Rev. D 65, 113002 (2002) hep-ph/0203224.

[15] F. Jegerlehner, hep-ph/0312372. For other values in the literature, see for example, K. Hagiwara, A. D. Martin, D. Nomura and T. Teubner, Phys. Rev. D 69, 093003 (2004) hep-ph/0312250; Preliminary result, H. Burkhardt and B. Pietrzyk (see talk by B. Pietrzyk at SigHad03 Pisa, 9 October 2003); M. Davier, S. Eidelman, A. Hocker and Z. Zhang, Eur. Phys. J. C 27, 497 (2003) hep-ph/0208177; R. Alemany, M. Davier and A. Hocker, Eur. Phys. J. C 2, 123 (1998) hep-ph/9703220.

[16] P. Azzi et al. [CDF Collaborattion], hep-ex/0404010.

[17] S. Eidelman et al. [Particle Data Group Collaboration], Phys. Lett. B 592, 1 (2004).

[18] M. E. Peskin and T. Takeuchi, Phys. Rev. D 46, 381 (1992).

[19] I. Maksymyk, C. P. Burgess and D. London, Phys. Rev. D 50, 529 (1994) hep-ph/9306267.

[20] H. E. Haber and G. L. Kane, Phys. Rept. 117, 75 (1985); S. P. Martin, hep-ph/9709356.

[21] S. P. Martin, Phys. Rev. D 66, 096001 (2002) hep-ph/0206136.

[22] A. Heister et al. [ALEPH Collaboration], Phys. Lett. B 533, 223 (2002) hep-ex/0203020.

[23] L. Randall and R. Sundrum, Nucl. Phys. B 557, 79 (1999); hep-th/9810155. G. F. Giudice, M. A. Luty, H. Murayama and R. Rattazzi, JHEP 9812, 027 (1998) hep-ph/9810442. 
[24] For example, the GigaZ option at a high-luminosity linear collider could measure $s_{\text {eff }}^{2}$ at the $10^{-5}$ level. For details, see R. Hawkings and K. Monig, Eur. Phys. J. directC 1, 8 (1999) hep-ex/9910022.

[25] G. Marandella, C. Schappacher and A. Strumia, hep-ph/0502095. 